\title{
Meralgia paresthetica affecting parturient women who underwent cesarean section
} -A case report-

\author{
Kum Hee Chung, Jong Yeon Lee, Tong Kyun Ko, Chung Hyun Park, Duk Hee Chun, Hyeon Jeong \\ Yang, Hyun Jue Gill, and Min Ku Kim \\ Department of Anesthesiology and Pain Medicine, CHA University School of Medicine, Seongnam, Korea
}

Meralgia paresthetica is commonly caused by a focal entrapment of lateral femoral cuteneous nerve while it passes the inguinal ligament. Common symptoms are paresthesias and numbness of the upper lateral thigh area. Pregnancy, tight cloths, obesity, position of surgery and the tumor in the retroperitoneal space could be causes of meralgia paresthetica. A 29-year-old female patient underwent an emergency cesarean section under spinal anesthesia without any problems. But two days after surgery, the patient complained numbness and paresthesia in anterolateral thigh area. Various neurological examinations and L-spine MRI images were all normal, but the symptoms persisted for a few days. Then, electromyogram and nerve conduction velocity test of the trunk and both legs were performed. Test results showed left lateral cutaneous nerve injury and meralgia paresthetica was diagnosed. Conservative treatment was implemented and the patient was free of symptoms after 1 month follow-up. (Korean J Anesthesiol 2010; 59: S86-S89)

Key Words: Cesarean section, Meralgia paresthetica, Spinal anesthesia.

Nueraxial anesthesia and regional anesthesia for Cesarean section are commonly practiced in obstetrics in the case of painless delivery due to recent dissemination of medical knowledge, simplicity of operation, and effective pain management. Especially, regional anesthesia is preferred for a woman who is going to deliver a baby and is having a complication from general anesthesia. In practicing regional anesthesia, symptoms such as infection, epidural hematoma, post-dural puncture headache, damage of nerves, and general poisoning due to intravenous injection of the anesthetic can occur. A woman delivering a baby can have nerve injury, along with anatomical and physiological changes, during her pregnancy [1-3]. When a woman has delivered a baby either through vaginal delivery or Cesarean section with regional anesthesia, and then complains of pain in the nervous system after childbirth, obstetricians refer this complaint to the anesthesia department. Thus, anesthetists have to determine whether the nerve damage occurring in the patient is due to the

Received: May 10, 2010. Revised: May 18, 2010. Accepted: June 23, 2010.

Corresponding author: Jong Yeon Lee, M.D., Department of Anesthesiology and Pain Medicine, CHA University School of Medicine, Yatapdong, Bundang-gu, Seongnam 463-712, Korea. Tel: 82-31-780-5557, Fax: 82-31-701-9433, E-mail: mdmax00@kornet.net

(c) This is an open-access article distributed under the terms of the Creative Commons Attribution Non-Commercial License (http:// creativecommons.org/licenses/by-nc/3.0/), which permits unrestricted non-commercial use, distribution, and reproduction in any medium, provided the original work is properly cited. 
anesthesia for Cesarean section or the pregnancy itself. We were referred to a case where a woman underwent Cesarean section with spinal anesthesia and complained of numbness in her left lateral thigh after the Cesarean section. The above-mentioned case was diagnosed as meralgia paresthetica. We present many cases of meralgia paresthetica, along with a literature review on meralgia paresthetica.

\section{Case Report}

A 29-year-old woman, being artificially inseminated with twins, was hospitalized to get a Cesarean section. A Cesarean section was scheduled for her on the next day, but sudden pain and progression of the childbirth delivery forced her to the childbirth delivery room early. As she had undergone a vaginal delivery 3 years ago, vaginal delivery was induced but failed. Therefore, a decision was made to do a Cesarean section.

After entering the operating room, the surgeons made the parturient woman take the lithotomy position to try a vaginal delivery again, but it was unsuccessful. Spinal anesthesia was administered to the patient since she had spent only 5 hours fasting. The surgeons put the patient in the right lateral decubitus position and disinfected the puncture part between the L3-L4 spinous processes with betadine solution. Then, the surgeons inserted 26 G Quinke, a spinal needle, into the puncture part by the midline approach method to verify whether the cerebrospinalfluid (CSF) would smoothly release. After seeing a smooth flow, the surgeons injected $10 \mathrm{mg}$ of hyperbaric $0.5 \%$ bupivacaine and $20 \mu \mathrm{g}$ of fentanyl. During the insertion of the needle and injection of the drug, the mother didn't complain of any pains or paresthesia on her back and lower limbs. Following the verification of the sensory blockade up to $\mathrm{T} 6$ thoracic vertebra 10 minutes after the drug injection, the surgeons began the operation for Cesarean section.

After the delivery, a Hartmann solution containing 20 units of oxytocin was injected; however, the vaginal contraction was still insufficient. Therefore, the surgeons injected $0.2 \mathrm{mg}$ of methylergometrine into her triceps brachialis muscle and $500 \mathrm{ml}$ of normal saline containing $1 \mathrm{mg}$ of sulprostone into her body at the rate of $100 \mathrm{ml}$ per hour. Thirty minutes after the delivery, when her blood pressure declined to $70 / 40 \mathrm{mmHg}$, $8 \mathrm{mg}$ of ephedrine was injected, and her blood pressure returned to the normal level and stayed at $110 / 60 \mathrm{mmHg}$ afterward. For the rest of the operation time, there was nothing unusual. Following the operation, the mother was brought to the postanesthetic recovery unit to be observed for 1 hour. After the doctors found that her sensory blockade was maintained up to T12 thoracic vertebra and there was no abnormality, they decided to move the patient to her ward. Two days after the operation, the anesthesiology department was asked for a joint treatment because the patient complained of numbness and paresthesia on her left leg. Although the mother said that she had paresthesia on her left femoral region, the doctors couldn't find any dyskinesia, decline in strength, lumbago, pooling of urine and feces, or paresthesia on the perineal region.

Furthermore, knee and ankle jerk reflexes were proven normal. The authors scanned her lumbar vertebrae with magnetic resonance imaging (MRI) to verify epidural hematoma or abscess, but nothing unusual was discovered. The authors also conducted a blood test to determine infection and inflammatory condition, and obtained the result that her white blood cell (WBC) count was $6,400 / \mu$ l and erythrocyte sedimentation rate (ESR) was $13 \mathrm{~mm} / \mathrm{hr}$, both of which are normal. In addition, she didn't suffer any fever. Subsequently, the authors decided to remain on the watch without additional treatment, considering that, according to neurological and laboratory tests, the patient had little possibility of nerve injury from spinal anesthesia, epidural hematoma, and abscess. However, after the patient continuously complained of the symptom for days, needle electromyography and nerve conduction studies were conducted on her truncus and lower limbs to identify any lesions in the nerve root and peripheral nerve.

As a result, the sensory nerve conduction velocity (SNCV) test discovered a left lateral cutaneous nerve injury while the needle electromyography study didn't find anything unusual (Fig. 1). In the following pelvic compression test, the patient said that her symptom was reduced when her left pelvis was compressed. Considering her symptom, which was limited to left lateral cutaneous nerve, and the result of the pelvic

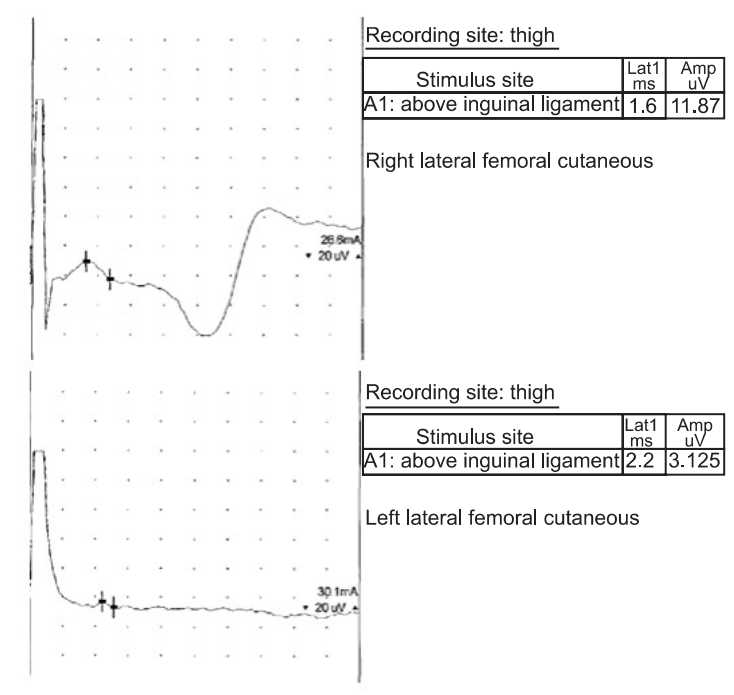

Fig. 1. Right and left lateral femoral cutaneous sensory nerve conduction velocity recording. The left lateral femoral cutaneous sensory nerve conduction amplitude is reduced. 
compression test, the authors diagnosed her as meralgia paresthetica and prescribed nonsteroidal anti-inflammatory drugs for conservative treatment. She was allowed discharge and was traced as an outpatient for a month. According to the result of the observance after discharge, the numbness on her left femoral region disappeared without special treatment, and complete blood cell count (CBC) and ESR were also within normal levels.

\section{Discussion}

During pregnancy, skeletal muscles and ligaments of parturient women become relaxed, and, with the progress of pregnancy, uteri expand and lordoses of lumbar vertebrae increase. For those reasons, parturient women are likely to suffer lumbago, pelvic pain, and radiculopathy $[3,4]$. In particular, the frequency of such neurotic complications escalates after deliveries or operation. This can be attributed to the following accidents in the process of delivery or operation: neurotmesis, damage in kidneys or blood vessels, or compression $[4,5]$.

Postpartum nerve injury often occurs to primiparas and is related to cephalopelvic disproportion and midforceps delivery. Especially, it happens more frequently if primiparas have prolonged pains during their second stage of labor. The nerve injury mainly results from endogenous factors, whereas direct damage caused by regional anesthesia is rare [4]. Generally, neuraxial anesthesia is greatly favored to reduce pain during labor or to conduct a Cesarean section. If parturient women show some neurotic symptoms after neuraxial anesthesia, doctors need to verify mass lesions, such as epidural hematoma or abscess. If diagnosis is delayed for more than 8 hours, the nerve injury can become permanent, thus early diagnosis and treatment is required [2]. In this research, 2 days after the operation, when the patient complained of paresthesia on her left femoral region, unaccompanied by any decline in strength and pain, the authors administered an MRI scan and blood test although epidural hematoma or abscess seemed unlikely. The test results showed there was nothing unusual. Also, the authors reckoned that the possibility of cauda equina syndrome was low since there was no pooling of urine and feces, sense deprivation on the perineal region, or declined strength of the lower limbs. Moreover, the author excluded the possibility of direct damage to spinal cords, cornus medullaris, and spinal nerve root because the patient didn't complain of any pain during the insertion of the spinal needle. Then, the authors conducted needle electromyography and nerve conduction studies on the patient's truncus and lower limbs since they suspected an endogenous nerve injury. As a result, the authors were able to exclude the possibility of radiculopathy since the needle electromyography showed a positive result. However, the nerve conduction study discovered a left lateral cutaneous nerve injury, leading the authors to diagnose meralgia paresthetica. After the series of tests, the patient fully recovered, along with conservative treatment and follow-up observation. The patient's such experience was also in line with the development of meralgia paresthetica.

Meralgia paresthetica, which affects 4.3 out of 10,000 people annually, is a kind of peripheral nerve disorder and is caused by mononeuropathy of the lateral femoral cutaneous nerve. This disease can be characterized by numbness limited to the front femoral region, weakened response to a pinprick, pain, or the sensation of burning fire. Generally, the symptom is temporary but hardly clear [6]. The lateral femoral cutaneous nerve stretches from under the anterior iliac spine to the under the inguinal ligament. A lot of entrapments happen in this part of body and result in meralgia paresthetica [7]. The culprits for this disorder are largely twofold: automatic and idiopathic reasons. The examples of the former are mechanical factors that increase the pressure inside the stomach, such as obesity, pregnancy, tight clothes and belts, and pelvic benign tumors. Meanwhile, diopathic reasons include pelvic osteotomy, orthopedic treatments including spinal surgeries, laparoscopic cholecystectomy, laparoscopic myomectomy, coronary artery bypass grafting surgery, aortic valve replacement surgery, and bariatric surgery for extreme obesity [8-10]. In this research, the authors thought that the meralgia paresthetica of the patient was caused by the increased intra-abdominal pressure due to pregnancy and labor, as well as the lithotomy position, which compressed the lateral femoral cutaneous nerve.

Meralgia paresthetica is diagnosed by physical examination such as a pelvic compression test, which determines whether specific clinical symptoms (e.g., numbness of front thigh and paresthesia) and symptoms of the patient are improved when she is laid in the lateral decubitus position and her pelvis is pressed. If the patient has symptoms in bilaterality or complains of dyskinesia, and abnormal senses lead to distinct pain, other diseases that may cause sense paresthesia, such as a herniated intervertebral disk of the lumbar vertebra or radiculopathy, should be detected. During a clinical examination in which it is difficult to clearly diagnosis the patient, the diagnosis can be confirmed by a neurophysiological test. It is known that almost all patients with meralgia paresthetica show abnormality in the sensory nerve conduction study of the lateral femoral cutaneous nerve. A neurophysiological test other than the sensory nerve conduction study, such as a somatosensory evoked potential, can be also helpful in diagnosis $[9,11,12]$.

In treatment, most patients with meralgia paresthetica fully recover (nearly about $85 \%$ ) within 4-6 months with conservative treatment. As for conservative treatment, nonsteroidal anti-inflammatory drugs, tricyclic antidepressants, 
and anticonvulsants can help ease symptoms. In this casuistics, only conservative treatment brought about an improvement in the symptoms in times of diagnosing meralgia paresthetica, but in the case of severe symptoms, 1) local nerve block therapy that uses $0.25 \%$ of bupivacaine and corticosteroid or 2) ultrasound guidance block therapy on the lateral femoral cutaneous nerve are known as effective. A local steroid reduces excessive irritability of neurons and C-fibers. If those nerve block therapies do not work - though this would be a rare case - then operational decompression of the inguinal ligament, neurolysis of the lateral femoral cutaneous nerve, or transposition are recommended $[8,13]$.

Nerve injury that the mother suffers after part of the regional anesthesia has been administered is not necessarily caused by the regional anesthesia but can be triggered by pregnancy, delivery, or the patient's position during operation. Permanent nerve injury related to delivery is rare. However, as temporary nerve injury is not infrequent, anesthesiologists should differentiate post-delivery nerve injury from injury caused by neuraxial anesthesia, in order to prevent that patient from developing complications that can result in permanent disability.

\section{References}

1. Wong CA. Neurologic deficits and labor analgesia. Reg Anesth Pain Med 2004; 29: 341-51.

2. Wlody D. Complications of regional anesthesia in obstetrics. Clin Obstet Gynecol 2003; 46: 667-78.
3. Mabie WC. Peripheral neuropathies during pregnancy. Clin Obstet Gynecol 2005; 48: 57-66.

4. Wong CA, Scavone BM, Dugan S, Smith JC, Prather H, Ganchiff $\mathrm{JN}$, et al. Incidence of postpartum lumbosacral spine and lower extremity nerve injuries. Obstet Gynecol 2003; 101: 279-88.

5. Birnbach DJ, Hernandez M, van Zundert AA. Neurologic compli cations of neuraxial analgesia for labor. Curr Opin Anaesthesiol 2005; 18: 513-7.

6. van Slobbe AM, Bohnen AM, Bernsen RM, Koes BW, BiermaZeinstra SM. Incidence rates and determinants in meralgia paresthetica in general practice. J Neurol 2004; 251: 294-7.

7. Jefferson D, Eames RA. Subclinical entrapment of the lateral femoral cutaneous nerve: an autopsy study. Muscle Nerve 1979; 2: 145-54.

8. Harney D, Patijn J. Meralgia paresthetica: diagnosis and management strategies. Pain Med 2007; 8: 669-77.

9. Cho KT, Lee HJ. Prone position-related meralgia paresthetica after lumbar spinal surgery: a case report and review of the literature. J Korean Neurosurg Soc 2008; 44: 392-5.

10. Peters G, Larner AJ. Meralgia paresthetica following gynecologic and obstetric surgery. Int J Gynaecol Obstet 2006; 95: 42-3.

11. Nouraei SA, Anand B, Spink G, O'Neill KS. A novel approach to the diagnosis and management of meralgia paresthetica. Neurosurgery 2007; 60: 696-700.

12. Cordato DJ, Yiannikas C, Stroud J, Halpern JP, Schwartz RS, Akbunar M, et al. Evoked potentials elicited by stimulation of the lateral and anterior femoral cutaneous nerves in meralgia paresthetica. Muscle Nerve 2004; 29: 139-42.

13. Tumber PS, Bhatia A, Chan VW. Ultrasound-guided lateral femoral cutaneous nerve block for meralgia paresthetica. Anesth Analg 2008; 106: 1021-2. 